\title{
EL ESPACIO DE RADON Y EL SEGUNDO TEOREMA DE THALES DE MILETO
}

Fecha de recepción: 19 de abril de 2014 • Fecha de aceptación: 20 de mayo de 2014

\section{RADON'S SPACE AND THE SECOND THALES OF MILETU'S THEOREM}

Ricardo J. de Armas Costa', Lucio Rojas Cortés ${ }^{2,4}$ Fabio H. Castellanos Moreno³

\section{RESUMEN}

En el contexto médico, después que un paciente ha sido irradiado con rayos-x, los detectores del tomógrafo registran los valores de la densidad correspondientes a un corte seleccionado de su cuerpo. La información obtenida en el dominio espacial (sistema de coordenadas del paciente) es representada por medio de unas funciones denominadas proyecciones en el dominio o espacio de Radon (sistema de coordenadas de la máquina de imágenes). Posteriormente, la imagen borrosa del espacio de Radon es reconstruida en el dominio espacial por medio de algoritmos de inversión de la transformada de Radon. Existe una variedad de algoritmos de reconstrucción de imágenes tomográficas; unos organizan las proyecciones en forma cartesiana y otros en forma polar. En este documento se describen los lugares geométricos generados por puntos del corte, tanto en el espacio cartesiano de Radon como en el espacio polar de Radon. Las trayectorias en el espacio polar de Radon son consecuencia del segundo Teorema de Thales de Mileto.

Palabras clave: Proyecciones, Transformada de Radon, Coordenadas Cartesianas, Coordenadas Polares, Sinograma.

1. Docente Investigador, Departamento de Matemáticas, Universidad Central.

2. Docente Investigador, Departamento de Matemáticas, Universidad Militar Nueva Granada, Universidad Central.

3. Docente Investigador, Departamento de Matemáticas, Universidad Central.

4. Autor corresponsal: lucio.rojas@unimilitar.edu.co 


\section{ABSTRACT}

In the medical context, after a patient has been irradiated with x-ray tomography detectors record the values of the density corresponding to a selected section of your body. The information obtained in the spatial domain (patient coordinate system) is represented by means of projections functions called the domain or Radon space (coordinate system machine images). Subsequently, the blur of the Radon space is reconstructed in the spatial domain by inversion algorithms Radon transform. A variety of algorithms for tomographic image reconstruction; organized projections about Cartesian form and others in polar form. This document loci generated cut points, both in Cartesian space Radon and Radon polar space are described. The trajectories in the polar space of Radon are the result of the second theorem of Thales of Miletus.

Keywords: Projections, Radon Transform, Cartesian Coordinates, Polar Coordinates, Sinogram.

\section{INTRODUCCIÓN}

Al leer el título de este artículo "El espacio de Radon y el segundo Teorema de Thales de Mileto" podrían surgir preguntas como: ¿Qué relación se puede establecer entre Thales de Mileto y Johann Radon? ¿Existe alguna relación entre el segundo Teorema de Thales de Mileto y la reconstrucción de imágenes tomográficas? entre otras.

Johann Radon, matemático, nació el 16 de diciembre de 1887 en Bohemia Austria, y murió el 25 de mayo de 1956 en Viena. En 1917 publicó un artículo donde demostraba que si se conocía la colección completa de las integrales de línea de una función en el plano, entonces se podía reconstruir dicha función, usando un operador definido por él; que ahora es conocido con el nombre de transformada de Radon (Radon, 1917; Cormack, 1963; Boman, 1990; Deans, 1983; Helgason, 1980; Natterer, 1986). Estos resultados se convertirían en los fundamentos matemáticos para la reconstrucción de imágenes tomográficas durante la era de la computación y después de la aparición del algoritmo de la Transformada
Rápida de Fourier. Radon, fue además un cantante talentoso con una hermosa voz de barítono que en algún momento pensó en abandonar su formación matemática para convertirse en cantante de ópera; tocaba el violín en un trío.

Thales de Mileto fue uno de los siete sabios de Grecia. El agua para él era el principio, sustancia y fundamento de todas las cosas. Algunas de sus frases célebres son: "Conócete a ti mismo", "Abstenerse de hacer lo que criticamos en los demás" y "La ignorancia es una pesada carga". Era de ascendencia fenicia, aunque no existen acuerdos sobre las fechas exactas de su existencia, lo que parece más probable es que habría nacido en el año 624 a.C. y fallecido en el 547 a.C. Poco se sabe de su vida e incluso de su obra. Se le reconoce como el primer matemático atribuyéndosele cinco teoremas geométricos y la solución de dos problemas prácticos. Además de matemático era filósofo y astrónomo (Collette, 1985; Farrinton, 1979; Heath, 1981; Rey y Babini, 1986; Thomas, 1967). 


\section{La mayoría de los algoritmos de reconstrucción de imágenes tomográficas implementados actualmente organizan las pro- yecciones en rejillas cartesianas. Esto ha permitido que el lugar geométrico del espacio cartesiano de Radon del corte seleccio- nado de un paciente haya sido estudiado ampliamente.}

La mayoría de los algoritmos de reconstrucción de imágenes tomográficas implementados actualmente organizan las proyecciones en rejillas cartesianas. Esto ha permitido que el lugar geométrico del espacio cartesiano de Radon del corte seleccionado de un paciente haya sido estudiado ampliamente (Davison y Grünbaum, 1981; Falconer, 1983; Gordon, 1975; Herman, 1980). Son pocos los trabajos que analizan el lugar geométrico que describen los puntos en el espacio polar de Radon (Buzug, 2008). Sin embargo, es importante desde una perspectiva matemática, realizar este tipo de trabajos dado que se pueden descubrir relaciones que posiblemente no han sido visualizadas, enriqueciendo el tratamiento de imágenes tomográficas. Este estudio permite relacionar el tratamiento de imágenes tomográficas con el segundo Teorema de Thales de Mileto que dice que si $P$ es un punto de la circunferencia de diámetro $P_{1} P_{2^{\prime}}$ distinto de $P_{1}$ y $P_{2^{\prime}}$ entonces el triángulo $P_{1} P P_{2}$ es un triángulo rectángulo.

\section{PARTE EXPERIMENTAL}

\section{Simulaciones}

Las simulaciones se realizaron con el software MatLab versión R2012b de la empresa MathWorks.

\section{El corte tomográfico}

Se simuló con una imagen fantasma de color negro con resolución 256 × 256 pixeles y con algunos puntos $\mathrm{P}(\mathrm{x}, \mathrm{y})$ de color blanco.

\section{El espacio cartesiano de Radon}

Para visualizar el espacio cartesiano de Radon y las trayectorias de los puntos $\mathrm{P}(\mathrm{x}, \mathrm{y})$ de color blanco se manejó la rutina de MatLab radon.

\section{El espacio polar de Radon}

Paravisualizarelespacio polar de Radon ylas trayectorias de los puntos $\mathrm{P}(\mathrm{x}, \mathrm{y})$ de color blanco se ajustó la referencia polar al código de la rutina de MatLab radon.

\section{RECONSTRUCCIÓN DE IMÁGENES TOMOGRÁFICAS}

Para comprender el proceso de la reconstrucción de imágenes tomográficas se necesita de conceptos como: sistemas de coordenadas, trayectoria de los rayos- $x$, muestreo de trayectorias, proyecciones, la transformada de Radon y algoritmos de inversión entre otros (Defrise y Guillberg, 2006; Kalender, 2006; Wang y Vannier, 1998; Herman, 2009; Natterer, 1985 y 1986; Gonzales y Wood, 2002; Bracewell, 1995; Cormack, 1963; Grinberg, 1985; Symeonidis, 1999; Shepp y Kruskal, 1978). 


\section{Los sistemas de coordenadas}

Se establecen dos sistemas de coordenadas: uno fijo para el paciente en reposo $x y$ y otro para el sistema de muestreo rotacional del tomógrafo st. La figura 1 ilustra los dos sistemas de coordenadas rotados con un ángulo $\theta$.

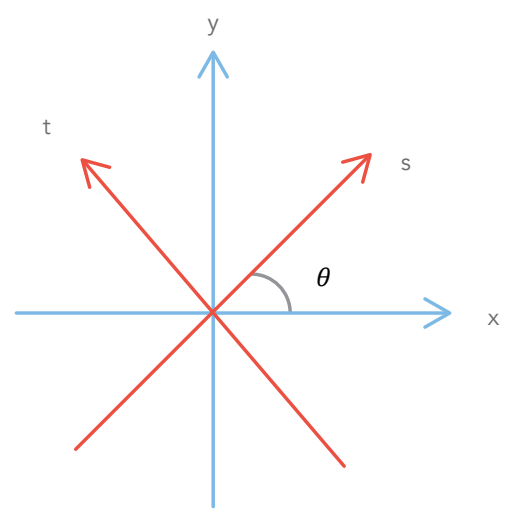

Figura 1. Sistema de coordenadas $\mathrm{xy}-\mathrm{st}$

\section{La trayectoria de los rayos-x}

Sea $\mathrm{f}(\mathrm{x}, \mathrm{y})$ la imagen del corte seleccionado de un paciente que será reconstruida. La expresión matemática de la trayectoria que siguen los rayos-x a través del corte en un escaneo de haces paralelos se modela a continuación. La figura 2 ilustra un rayo-x en la dirección de $\overrightarrow{\mathrm{AB}}$ y perpendicular al eje s.

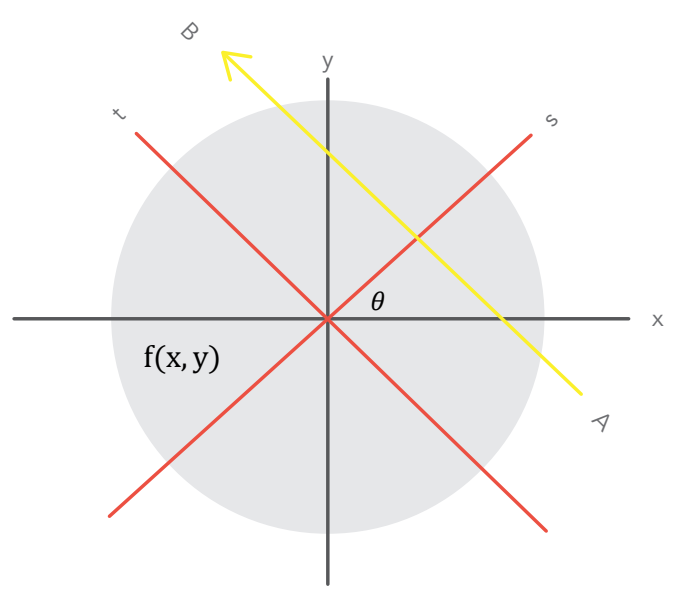

Figura 2. Rayo $\overrightarrow{\mathrm{AB}}$ atravesando el corte.
En la figura 3 se dibujan los triángulos rectángulos $\triangle \mathrm{OPC}$ y $\triangle \mathrm{OPD}$.

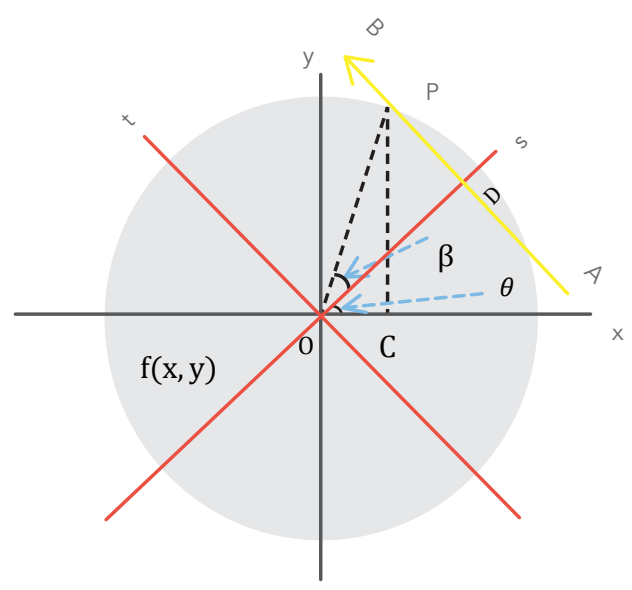

Figura 3. Triángulos rectángulos $\triangle \mathrm{OPC}$ y $\triangle \mathrm{OPD}$

De los triángulos $\triangle \mathrm{OPC}$ y $\triangle \mathrm{OPD}$ se obtiene:

$$
\begin{aligned}
& \overline{\mathrm{OP}} \cos (\theta+\beta)=\mathrm{x} \\
& \overline{\mathrm{OP}} \operatorname{sen}(\theta+\beta)=\mathrm{y} \\
& \overline{\mathrm{OP}} \cos (\beta)=\mathrm{s} \\
& \overline{\mathrm{OP}} \operatorname{sen}(\beta)=\mathrm{t}
\end{aligned}
$$

Se aplica la identidad para la suma de ángulos en (1) y (2).

$$
\begin{array}{ll}
\overline{\mathrm{OP}} \cos \theta \cos \beta-\overline{\mathrm{OP}} \operatorname{sen} \theta \operatorname{sen} \beta=\mathrm{x} & (5) \\
\overline{\mathrm{OP}} \cos \theta \operatorname{sen} \beta+\overline{\mathrm{OP}} \operatorname{sen} \theta \cos \beta=y
\end{array}
$$

Sustituyendo (3) y (4) en (5) y (6) respectivamente, se obtienen las expresiones que relacionan los dos sistemas de coordenadas y el ángulo $\theta$ de rotación.

$$
\begin{aligned}
& (s) \cos \theta-(t) \operatorname{sen} \theta=x \\
& (t) \cos \theta+(s) \operatorname{sen} \theta=y
\end{aligned}
$$

Cuando los sistemas de coordenadas $x y$ y st satisfacen (7) y (8) se dice que están relacionados por una rotación de ejes. Para expresar $S$ en términos de 
$\mathrm{x}, \mathrm{y}, \theta$ se multiplica (7) por el $\cos \theta$ y (8) por el $\operatorname{sen} \theta$. Después se suman las expresiones multiplicadas.

$$
s=x \cos \theta+y \operatorname{sen} \theta \quad(9)
$$

De manera similar para expresar ' $t$ en términos de $\mathrm{x}, \mathrm{y}, \theta$ se multiplica (7) por el $\operatorname{sen} \theta$ y (8) por el $\cos \theta$. Después se suman las expresiones multiplicadas.

La trayectoria del rayo-x a través del corte es descrita por la ecuación (9) como se precia en la figura 4.

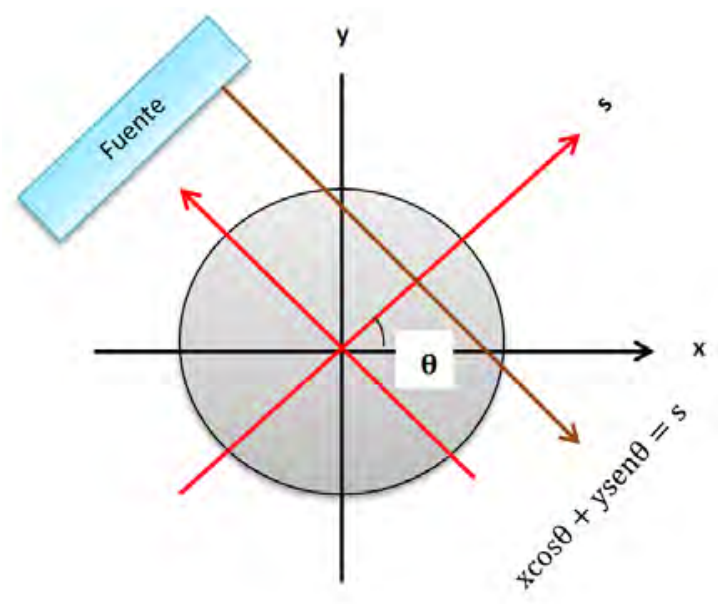

Figura 4. Ecuación de la trayectoria del rayo-x.

\section{Muestreo de los rayos-x}

Para muestrear los rayos-x que atraviesan el corte seleccionado del paciente se usa: la distribución delta Dirac, el operador de convolución y (9).

$$
f \star \delta(s)=\int_{-\infty}^{+\infty} \int_{-\infty}^{+\infty} f(x, y) \delta(x \cos \theta+y \operatorname{sen} \theta-s) d x d y
$$

\section{Proyecciones}

Al conjunto de rayos-x que atraviesan el corte en una dirección específica $\theta$ lo denominaremos proyección o perfil de atenuación y lo denotaremos con $\mathrm{p}_{\theta}(\mathrm{s})$.

$$
p_{\theta}(s)=\int_{-\infty}^{+\infty} \int_{-\infty}^{+\infty} f(x, y) \delta(x \cos \theta+y \operatorname{sen} \theta-s) d x d y
$$

En la figura 5 se presentan dos proyecciones tomadas para los ángulos $\mathrm{p}_{\theta=0^{\circ}}$ y $\mathrm{p}_{\theta=90^{\circ}}$.

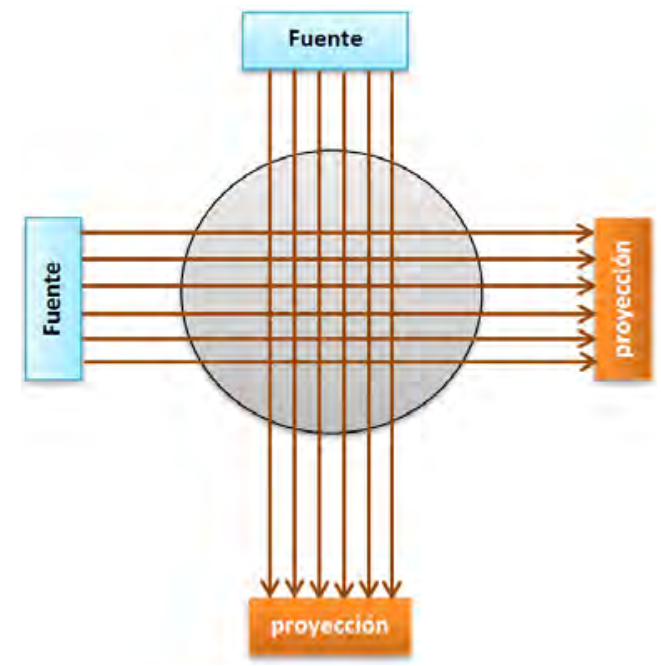

Figura 5. Proyecciones $\mathrm{p}_{\theta=0^{\circ}}$ y $\mathrm{p}_{\theta=90^{\circ}}$

\section{La transformada de Radon bidimensional}

La transformada de Radon de una función $\mathrm{f}(\mathrm{x}, \mathrm{y})$ definida en el plano es la integral de sobre un conjunto de rectas. Para las rectas dadas por la ecuación (9), la transformada de Radon se expresa como:

$$
\mathcal{R}[\mathrm{f}](\theta, \mathrm{s})=\int_{-\infty}^{+\infty} \int_{-\infty}^{+\infty} \mathrm{f}(\mathrm{x}, \mathrm{y}) \delta(\mathrm{x} \cos \theta+\mathrm{ysen} \theta-\mathrm{s}) \mathrm{dxdy}(12)
$$

La transformada de Radon aplicada a la tomografía computarizada convencional corresponde al conjunto de todas las proyecciones obtenidas del corte.

$$
\mathcal{R}[\mathrm{f}](\theta, \mathrm{s})=\mathrm{p}_{\theta}(\mathrm{s}) \quad(13)
$$

El espacio $(\theta, s)$ se denomina espacio de Radon. La transformada de Radon mapea el dominio espacial $(\mathrm{x}, \mathrm{y})$ hasta el dominio de Radon $(\theta, \mathrm{s})$. 


\section{Algoritmos de inversión}

Para reconstruir la imagen $\mathrm{f}(\mathrm{x}, \mathrm{y})$ del corte a partir del conjunto de sus proyecciones $\mathrm{p}_{\theta}(\mathrm{s})$ se debe computar la transformada inversa de Radon. Existen diversos algoritmos que son computacionalmente eficientes para invertir la transformada de Radon bidimensional. En la literatura de reconstrucción computarizada de imágenes tomográficas se encuentran una variedad de métodos: desde los métodos analíticos hasta los iterativos (Markoe, 1986; Smith, 1984; Quinto, 1980; Macovski, 1983; Ludwig, 1966; Nievergelt, 1986; Smith, 1984). De acuerdo con el algoritmo de reconstrucción implementado, el espacio de Radon se arregla en una rejilla cartesiana o en una rejilla polar.

\section{EL ESPACIO CARTESIANO DE RADON}

La mayoría de las veces las proyecciones $\mathrm{p}_{\theta}(\mathrm{s}) \mathrm{se}$ organizan en una rejilla cartesiana. En la figura 6b) las filas nos indican los valores de las integrales de línea registrados por los detectores y las columnas hacen referencia a los ángulos de cada proyección. Los valores de las proyecciones son de naturaleza discreta y forman el espacio de Radon del objeto siendo adquiridos en un rango de $0^{\circ}$ hasta $179^{\circ}$ porque las trayectorias de rayos-x por detrás del paciente examinado no proporcionan información diferente a las trayectorias ya medidas.

\section{Lugar geométrico}

Las coordenadas polares del punto $\mathrm{P}(\mathrm{x}, \mathrm{y})$ de color blanco se indican en la figura 7.

$$
\begin{aligned}
& x=r \cos \phi \\
& y=r \operatorname{sen} \phi
\end{aligned}
$$

a) Espacio del paciente

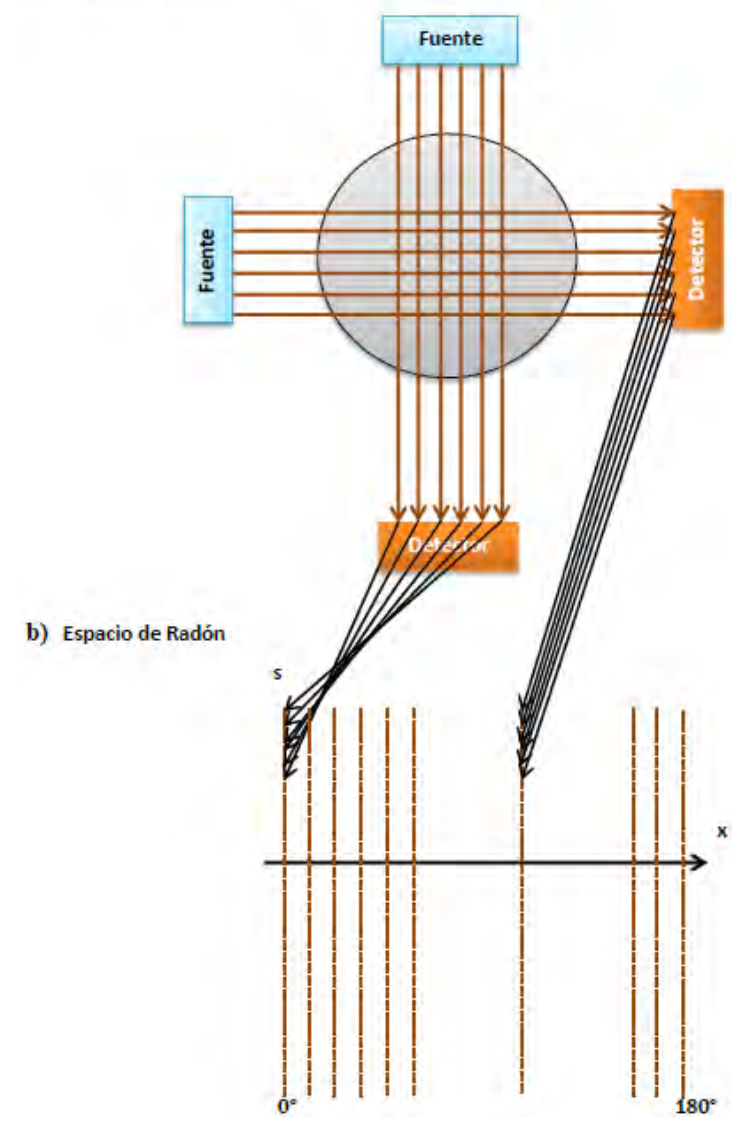

Figura 6. a) Valores medidos de las proyecciones en el espacio del objeto $(x, y)$ b) Arreglo cartesiano de los valores de las proyecciones en el espacio de Radon $(s, \theta)$.

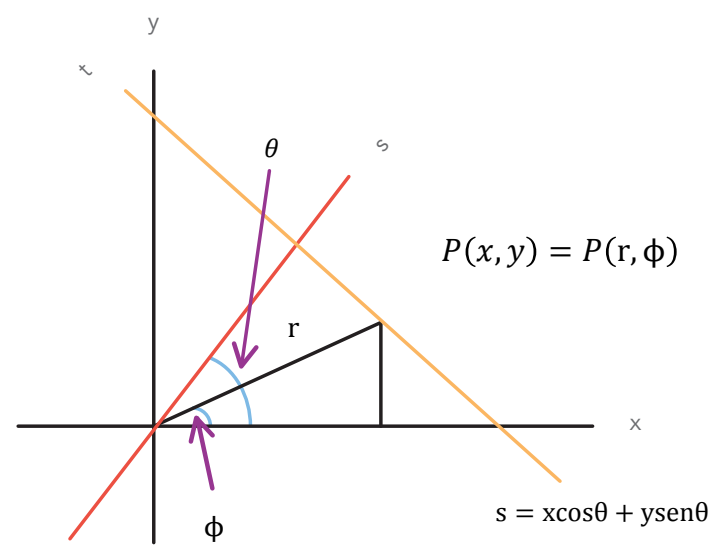

Figura 7. Coordenadas polares del punto $P(x, y)$ 


$$
\begin{aligned}
& \mathrm{s}=(\mathrm{r} \cos \phi) \cos \theta+(\mathrm{rsen} \phi) \operatorname{sen} \theta(16) \\
& \mathrm{s}=\mathrm{r} \cos (\theta-\phi)
\end{aligned}
$$

El lugar geométrico de $\mathrm{P}(\mathrm{x}, \mathrm{y})$ en el espacio cartesiano de Radon viene dado por (17). Este es un espacio de sinusoides que se le denomina el sinograma.

\section{Simulación}

En la figura 8 se visualizan las trayectorias generadas por los puntos de color blanco en el espacio cartesiano de Radon.

\section{EL ESPACIO POLAR DE RADON}

Las proyecciones $\mathrm{p}_{\theta}(\mathrm{s})$ se organizan en una rejiIla polar como lo muestra la figura 9.

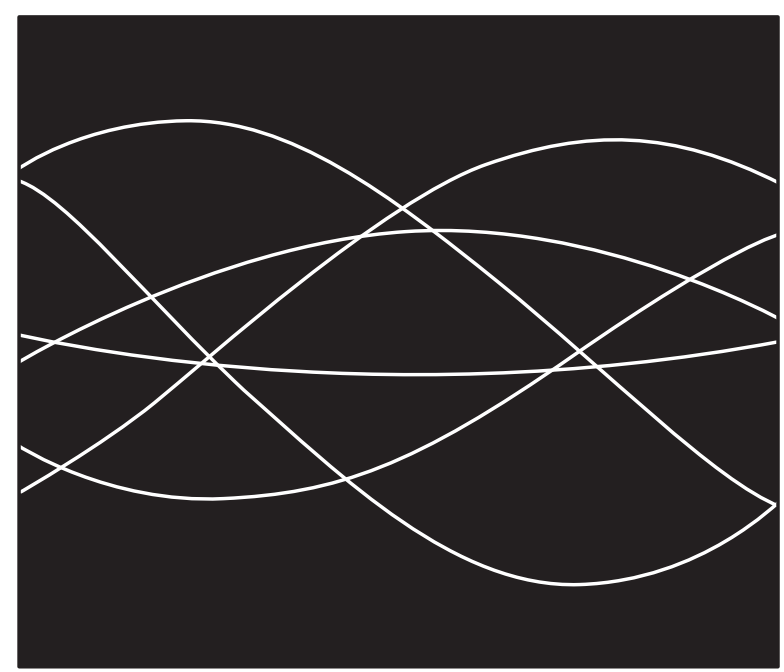

Figura 8. Trayectoria de los puntos de color blanco en el espacio Cartesiano de Radon

ISSN 1900-4699 • Volumen 10 • Número 1 • Páginas 56-65 • 2014

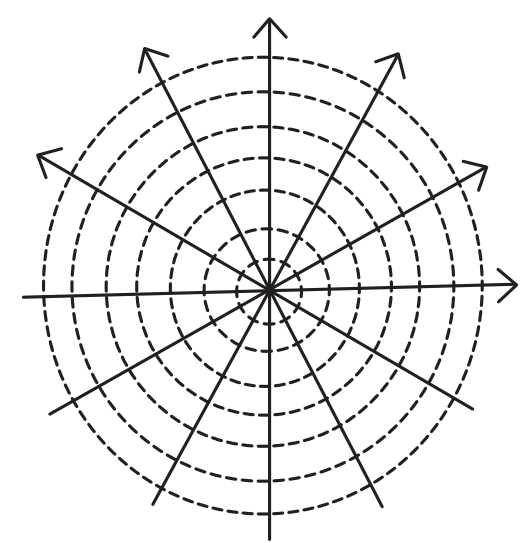

Figura 9. Arreglo en forma polar de las proyecciones.

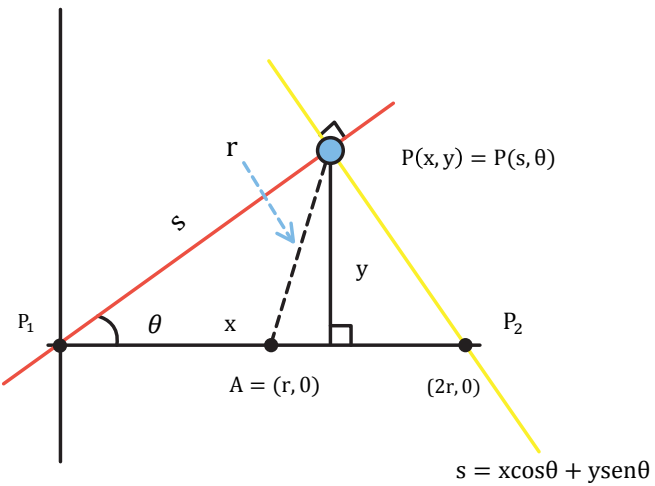

Figura 10. Coordenadas polares de la partícula

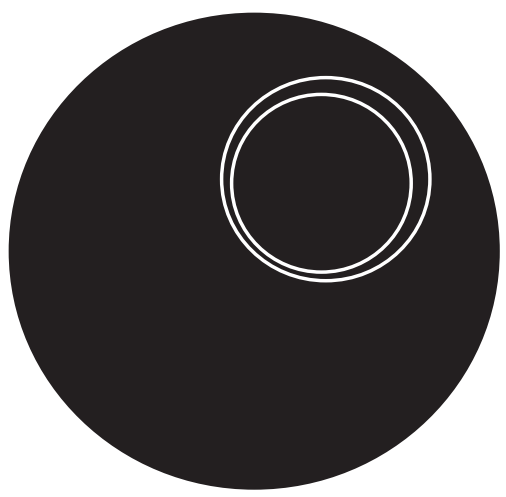

Figura 11. Trayectorias de los puntos de color blanco en el espacio Polar de Radon 


\section{Cuando el conjunto de las proyecciones tomadas del corte de un} paciente se arreglan en forma cartesiana, un punto de la imagen describe una trayectoria sinusoide en el espacio de Radon; si las proyecciones se arreglan en forma polar, un punto de la imagen describe una trayectoria circular.

\section{Lugar geométrico}

Las proyecciones que contienen la información del punto $\mathrm{P}(\mathrm{x}, \mathrm{y})$ generan un patrón de triángulos (Buzug, 2008). Las coordenadas polares del punto $\mathrm{P}(\mathrm{x}, \mathrm{y})$ se indican en la figura 10. Si $\mathrm{P}$ es un punto de la circunferencia de diámetro $\mathrm{P}_{1} \mathrm{P}_{2}$ entonces por el segundo Teorema de Thales de Mileto el triángulo $\mathrm{P}_{1} \mathrm{PP}_{2}$ es rectángulo. Es decir, el patrón generado es de triángulos rectángulos.

$$
\begin{aligned}
& x=(s) \cos \theta \\
& y=(18) \operatorname{sen} \theta
\end{aligned}
$$

Las distancias $d\left(P_{1}, A\right)$ y $d(A, P)$ son iguales, por tanto:

$$
x^{2}+y^{2}=2 r x
$$

Se sustituyen (18) y (19) en (20).

$$
\begin{aligned}
& ((s) \cos \theta)^{2}+((s) \operatorname{sen} \theta)^{2}=2 r((s) \cos \theta) \\
& s=2 r \cos \theta
\end{aligned}
$$

El lugar geométrico del punto $\mathrm{P}(\mathrm{x}, \mathrm{y})$ en el espacio polar de Radon viene dado por (22). Este es un espacio de círculos (Buzug, 2008).

\section{Simulación}

En la figura 11 se visualizan las trayectorias generadas por los puntos de color blanco.

\section{CONCLUSIONES}

Cuando el conjunto de las proyecciones tomadas del corte de un paciente se arreglan en forma cartesiana, un punto de la imagen describe una trayectoria sinusoide en el espacio de Radon; si las proyecciones se arreglan en forma polar, un punto de la imagen describe una trayectoria circular. Estas trayectorias circulares en el espacio polar de Radon son consecuencia del segundo Teorema de Thales de Mileto. 


\section{REFERENCIAS}

1. Boman J. 1990. On generalized Radon transforms with unknown measures, Contemporary Mathematics, 113:5-15.

2. Bracewell R.N. 1995. Two-dimensional imaging. Eglewood Cliffs, NJ: Prentice-Hall.

3. Buzug T.M. 2008. Computed Tomography. From Photon Statistics to Modern Cone Beam CT. Leipzig, Germany: Springer.

4. Cormack A. M. 1963. Representation of a function by its line integrals, with some radiological applications. Journal of Applied Physics, 34:2722-2727.

5. Collette J. P. 1985. Historia de las matemáticas, Vol. I. Madrid: Siglo XXI.

6. Donson M. E., y Grünbaum F.A. 1981. Tomographic reconstructions with arbitrary directions. Comm. Pure and Applied Mathematics, 34:77-120.

7. Deans S. R. 1983. The Radon Transform and some of its applications. New York: John Wiley and Sons Inc.

8. Defrise M., y Guillberg G. T. 2006. Image Reconstruction. Physics in Medicine and Biology, 51:139-154.

9. Farrington B. 1979. Ciencia Griega. Barcelona: Icaria.

10. Falconer K. J. 1983. X-ray problems for point sources. Proceedings London Mathematical Society, 46:241-262.
11. Gordon R. 1975. Image processing for 2-D and $3-D$ reconstruction from projections: theory and practice in medicine and the physical sciences. Digest of technical papers, Stanford, California, August 4-7 (1975), Sponsored by the Optical Society of America.

12. Grinberg E. 1985. On images of Radon transforms. Duke Mathematical Journal, 52:939-972.

13. Gonzáles R.C., y Woods R.E. (2002). Digital Image Processing. New Jersey: Prentice-Hall.

14. Helgason S. 1980. The Radon Transform. Birkhäuser. Second Edition.

15. Herman, G. T. 1980. Image Reconstruction from Projections. The Fundamentals of Computarized Tomography. Academic Press.

16. Health T. 1981. A history of greek mathematics, Vol. I. New York: Dover.

17. Herman G.T. 2009. Fundamentals of Computarized Tomography: Image Reconstruction from Projections (Advances in Pattern Recognition). 2nd Ed, Springer.

18. Kak A. C., y Sallaney M. 1988. Principles of Computarized Tomography". New York: IEEE press.

19. Kalender W. A. 2006. Computed Tomography: Fundamentals, System Technology, Image Quality, Apllications. Wiley. 
20. Ludwig D. 1966. The Radon transform on Euclidean Space. Communications on Pure and Applied Mathematics, 23:49-81.

21. Macovski A. 1983. Medical imaging systems. Englewood Cliffs, NJ: Prentice- Hall.

22. Markoe A. 1986. Fourier inversion of the attenuated Radon transform. SIAM: SIAM Journal on Mathematical Analysis, to appear.

23. Natterer F. 1985. Fourier reconstruction in tomography. Numerische Mathematik, 47:343-353.

24. Matterer F. 1986. The Mathematics of Computarized Tomography". New York: John Wiley\&Sons.

25. Nievergelt Y. 1986. Elementary inversion of Radon's transform. SIAM Reviews, 28:79-84.

26. Quinto E. T. 1980. The dependence of the generalized Radon transform defining measures. Transactions of the American Mathematical Society, 257:331-346.

27. Radon J. 1917. Über die Bestimmung von Funktionen durch ihre Ihre Integralwerte längs gewisser Mannigfaltigkeiten. Berichte Sächsische Akademie der Wissenschaften, Leipzig, Math-Phys. 69:262-277.

28. Radon J. 1986. On the Determination of Functions from Their Integral Values along Certain Manifolds. IEEE Transactions on Medical Imaging, 5:170-176.
29. Rey J., y Babini J. 1986. Historia de la matemática, Vol. I. Barcelona: Gedisa.

30. Shepp L. A., y Kruskal J.B. 1978. Computerized tomography: The new medical $X$ - ray technology. The American Mathematical Monthly, 85:420-438.

31. Smith K.T. 1983. Reconstruction formulas in computed tomography. Proceedings of Symposia in Applied Mathematics (AMS), 27:7-23.

32. Smith K.T. 1984. Inversion of the X-ray transform. SIAM-AMS Proceedings, 14:41-52.

33. Symeonidis E. 1999. On the image of a generalized d-plane transform on $\mathbb{R}^{n}$. Journal of Lie Theory, 9:39-68.

34. Thomas I. 1967. Greek Mathematics, Vol. I. London: W. Heinemann and Harvard Univ.

35. Wang G., y Vannier M. W. 1998. Computarized Tomography. Departament of Radiology. University of lowa. Iowa 52242, lowa City (USA). 